\title{
Molecular Mechanisms of Same TCM Syndrome for Different Diseases and Different TCM Syndrome for Same Disease in Chronic Hepatitis B and Liver Cirrhosis
}

\author{
Zhizhong Guo, ${ }^{1}$ Shuhao Yu, ${ }^{2}$ Yan Guan, ${ }^{1}$ Ying-Ya Li, ${ }^{1}$ Yi-Yu Lu, ${ }^{1}$ Hui Zhang, ${ }^{1}$ and Shi-Bing Su${ }^{1}$ \\ ${ }^{1}$ Research Center for Complex System of Traditional Chinese Medicine, Shanghai University of Traditional Chinese Medicine, \\ 1200 Cailun Road, Shanghai 201203, China \\ ${ }^{2}$ College of Life Science and Biotechnology, Shanghai Jiaotong University, 800 Dongchuan Road, Shanghai 200240, China
}

Correspondence should be addressed to Shi-Bing Su, shibingsu07@163.com

Received 9 February 2012; Revised 2 April 2012; Accepted 5 April 2012

Academic Editor: Aiping Lu

Copyright (C) 2012 Zhizhong Guo et al. This is an open access article distributed under the Creative Commons Attribution License, which permits unrestricted use, distribution, and reproduction in any medium, provided the original work is properly cited.

\begin{abstract}
Traditional Chinese medicine (TCM) treatment is based on the traditional diagnose method to distinguish the TCM syndrome, not the disease. So there is a phenomenon in the relationship between TCM syndrome and disease, called Same TCM Syndrome for Different Diseases and Different TCM Syndrome for Same Disease. In this study, we demonstrated the molecular mechanisms of this phenomenon using the microarray samples of liver-gallbladder dampness-heat syndrome (LGDHS) and liver depression and spleen deficiency syndrome (LDSDS) in the chronic hepatitis B (CHB) and liver cirrhosis (LC). The results showed that the difference between $\mathrm{CHB}$ and LC was gene expression level and the difference between LGDHS and LDSDS was gene coexpression in the G-protein-coupled receptor protein-signaling pathway. Therein genes GPER, PTHR1, GPR173, and SSTR1 were coexpressed in LDSDS, but not in LGDHS. Either CHB or LC was divided into the alternative LGDHS and LDSDS by the gene correlation, which reveals the molecular feature of Different TCM Syndrome for Same Disease. The alternatives LGDHS and LDSDS were divided into either CHB or LC by the gene expression level, which reveals the molecular feature of Same TCM Syndrome for Different Diseases.
\end{abstract}

\section{Introduction}

Traditional Chinese medicine (TCM) is a medical system with at least 3000 years of uninterrupted clinical practice in China. The TCM practice usually requires a TCM syndrome identification based on clinical manifestation followed by the use of individualized treatment that is adapted to address the particular TCM syndrome in patient [1]. Therefore, TCM syndrome, also called ZHENG or TCM pattern, is the core of diagnosis and treatment in TCM [2]. Nowadays, TCM syndrome had been studied in some specific disease such as hypertension [3], coronary heart disease [4], and rheumatoid arthritis [5] or biomedical condition such as neuroendocrine-immune network [6], suggesting that TCM syndromes are significantly associated with diseases.

Hepatitis B is a viral infection that attacks the liver and can cause both acute and chronic disease. Beyond 25\% of hepatitis B virus-infected patients would die of severe chronic liver diseases such as liver cirrhosis and liver cancer [7]. Chronic hepatitis B (CHB) and liver cirrhosis (LC) are the intractable diseases that remain a major public health problem worldwide. Although several antiviral drugs had been approved for $\mathrm{CHB}$, they caused significant side effects and drug resistance. In contrast, TCM treatment was regarded as a safe and effective method for $\mathrm{CHB}$ and Liver fibrosis $[8,9]$.

TCM treatment is based on the traditional diagnose method to differentiate the TCM syndrome, not the disease in western medicine. Therefore, TCM syndromes could be classified in CHB as well as in LC. Moreover, different patients, respectively, suffering CHB or LC could also belong to the same TCM syndrome. This phenomenon is called Same TCM Syndrome for Different Diseases and Different TCM syndrome for Same Disease [10-12]. This viewpoint in TCM is very different with Western medicine. The molecular mechanism of this phenomenon is still a mystery. 
Previous study reported liver-gallbladder dampnessheat syndrome (LGDHS) and liver depression and spleen deficiency syndrome (LDSDS) are the major syndromes in CHB $[13,14]$. In this study, the aim is to demonstrate the molecular mechanism of Same TCM Syndrome for Different Diseases and Different TCM Syndrome for Same Disease by the analysis of whole gene expression in the same syndrome as LGDHS or LDSDS of different diseases as CHB and LC and the same disease as CHB or LC of different syndromes as LGDHS and LDSDS.

\section{Material and Methods}

2.1. Samples. Blood samples from 92 patients were obtained. Therein 14 samples from 2 LGDHS and 3 LDSDS in CHB patients, 3 LGDHS and 3 LDSDS in LC patients and 3 healthy peoples were used to microarray test, and 78 samples from 20 LGDHS and 18 LDSDS in CHB patients, and 21 LGDHS and 19 LDSDS in LC patients were used to test and verify the accuracy of the result. All patients were from Shanghai Longhua Hospital and have signed an agreement with us. The blood samples were morning fasting venous blood and saved in $-20^{\circ} \mathrm{C}$ with $150 \mu \mathrm{L}$ EDTA.

2.2. RNA Extraction and Microarrays. Total RNA of leukocyte from the whole blood was extracted using TRIzol Reagent (Invitrogen, Carlsbad, CA, USA), and a quality control was carried out with NanoDrop ND-1000. The cDNAs were synthesized by the Invitrogen First-Strand cDNA Synthesis kits (Invitrogen, Carlsbad, CA, USA), and RNA polymerase was added to degrade RNA. The cDNA was labeled and hybridized using NimbleGen Homo sapiens 12x135K Arrays (Roche NimbleGen, Madison, WI, USA), according to the manufacturer's protocol.

2.3. Real-Time RT-PCR. Difference-expressed mRNAs were verified by real-time RT-PCR according to SYBR Green Realtime PCR Master Mix kit (TOYOBO, Osaka, Japan) manufacturer. The primer sequences were F: TGGTGTGCGCAGCCATCGTG, R: GCCAGTAACCGGCCACCTCG for DRD5; F: GCTCTGTCAGGGCTCAACCTCC, R: GGCACAAACTTGGAGAGACCGAGC for GABRA; F: GCTACGTGGCCGTGGTGCAT, R: CCGCGGTGCGAGAGAAGACC for SSTR1; F: AGCGAACCCCTCCCACCACA, R: CAGGAAGGCTTGGCTCCGGC for NPFF. F: ACAGAGCCTCGCCTTTGCCG, R: ACATGCCGGAGCCGTTGTCG for ACTB.

2.4. Microarray Data Preprocessing and Statistic Analysis. Microarray data preprocessing was performed using the GenePix software. Raw expression data were $\log 2$ transformed and normalized by quantile normalization. Probes were considered robustly expressed if Signal/Noise (SNR) $<2$.

We took the average of 3 healthy people in every probe and let every patient sample ratio be this average in every probe. In all the following pages: $\mathrm{CHB}$ means chronic hepatitis B versus normal; LC means liver cirrhosis versus normal; LGDHS means liver-gallbladder dampness-heat syndrome versus normal; LDSDS means spleen deficiency syndrome versus normal.

The $t$-test function in $\mathrm{R}$ software was used to select difference expressed gene (threshold: $P$ value $<0.01$ or $P$ value $<0.05)$ in diseases between CHB and LC as well as in TCM syndromes between LGDHS and LDSDS. GO enrichment analysis was executed using the selected genes.

Heatmap analysis, also executed in R, was computing the hierarchical clustering in both rows and columns according to the set of gene values and drawing a color image as a visible result.

The correlation analysis was used to analyze the correlation of difference expressed genes between CHB and LC or LGDHS and LDSDS. The level of significance was set at correlation coefficient $>0.5$.

2.5. Gene Module Analysis and Difference Coexpression Analysis. The Weighted Correlation Network Analysis (WGCNA) $\mathrm{R}$ package was used to run the gene module analysis (parameter: networkType $=$ signed, detectCutHeight $=0.97$ ) WGCNA was a systems biology method to describe the correlation patterns among genes across microarray samples. It was used to find clusters (modules) of highly correlated genes and summarizing the clusters using the Module Eigengene (ME) [15].

Furthermore, coXpress $\mathrm{R}$ package was used to analyze the difference coexpression (parameter: $s=$ pearson, $m=$ average, $h=0.4$ ). coXpress as a tool has been applied to identify groups of genes that display differential coexpression patterns in microarray datasets and its utility [16].

\section{Results and Discussion}

3.1. Difference Expression Analysis. At first, to find whether there were some significant genes that could characterize the difference between two disease and two TCM syndromes, $t$-test was used to select difference expression gene in both disease and TCM syndrome levels. The threshold was $P$ value less than 0.01 . Remarkably, 6579 in all 14352 genes were differentially expressed between CHB and LC, suggested that the difference in mRNA expression level was very clear, according to $\mathrm{CHB}$ and LC that were completely different diseases. In contrast, only 98 genes were differentially expressed between LGDHS and LDSDS. The heatmap of the 98 genes between LGDHS and LDSDS was showed in Figure 1. Moreover, though these genes were obviously differentiated into two syndromes, the 98 genes were in disorder, no significantly related function was found by GO enrichment analysis. It also was tried to change the threshold as $P$ value less than 0.05 and got 830 genes, but still any significantly related GO function was not found.

3.2. Gene Modules Related with Disease or TCM Syndrome. Due to the above result that the molecular mechanisms of the difference between two TCM syndromes could be not commendably explained with the single-gene difference expression method, then the gene module method was used to demonstrate the difference between diseases and TCM 


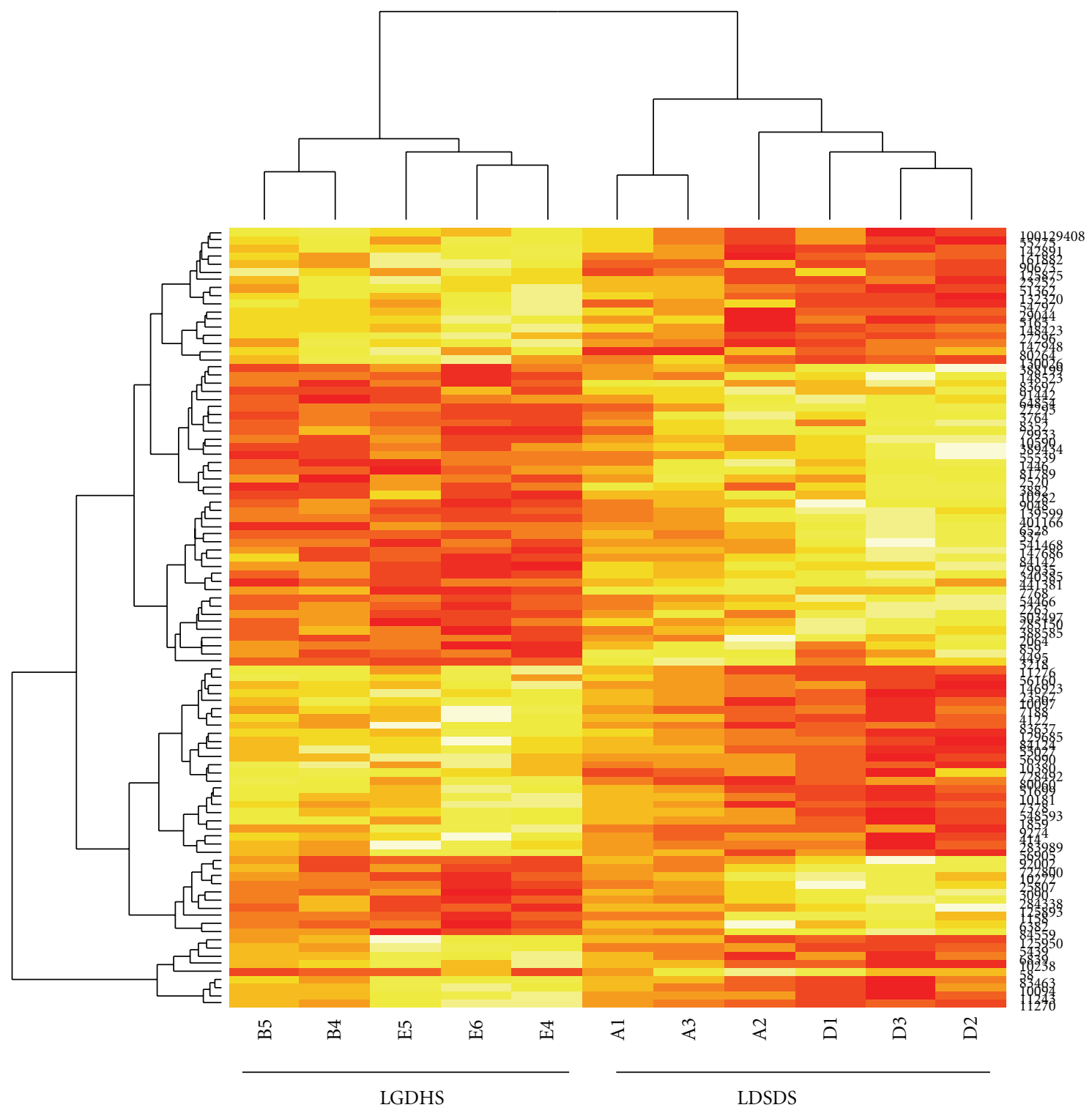

FIGURE 1: Heatmap of 98 differentially expressed genes between LGDHS and LDSDS. The 98 differentially expressed genes between LGDHS and LDSDS were obviously divided out by Heatmap analysis. Row: genes; column: patient number; deep colour: upexpressed genes; light colour: down-expressed genes; A1-3 and D1-3: LDSDS; B 4, 5 and E4-6: LGDHS.

syndromes. The all 14352 genes were taken into 26 gene modules by the WGCNA R package [15], and each module had a name of color and a ME to identify the gene expression. Among the 26 modules, some significant modules were screened out by correlating the MEs in our disease trail or TCM syndrome trail. In the result, blue, brown, turquoise, and yellow modules were most related with the difference between CHB and LC (Figure 2(a)), and lightgreen module and lightcyan module were most related with the difference between LGDHS and LDSDS (Figure 2(b)).

The above 6 gene modules were used to GO enrichment analysis. The result showed that the blue module was mainly enriched in G-protein-coupled receptor protein-signaling pathway, brown module was mainly enriched in immune system process, yellow module was mainly enriched in cell cycle, and turquoise module was enriched in many basal metabolisms. But it was still hard to understand that ossification function was enriched in lightcyan module, and the lightgreen module did not enrich in any GO function module.

\subsection{Comparing Difference Coexpression Network between Two} TCM Syndromes. To further demonstrate the mechanism of difference between two TCM syndromes, the correlation of gene expression including difference expression and difference coexpression was analyzed. Figure 3 was a schematic diagram which showed the meaning of difference expression or difference coexpression, respectively. The difference expression meant that there were gene different expression levels between two states. The difference coexpression meant 


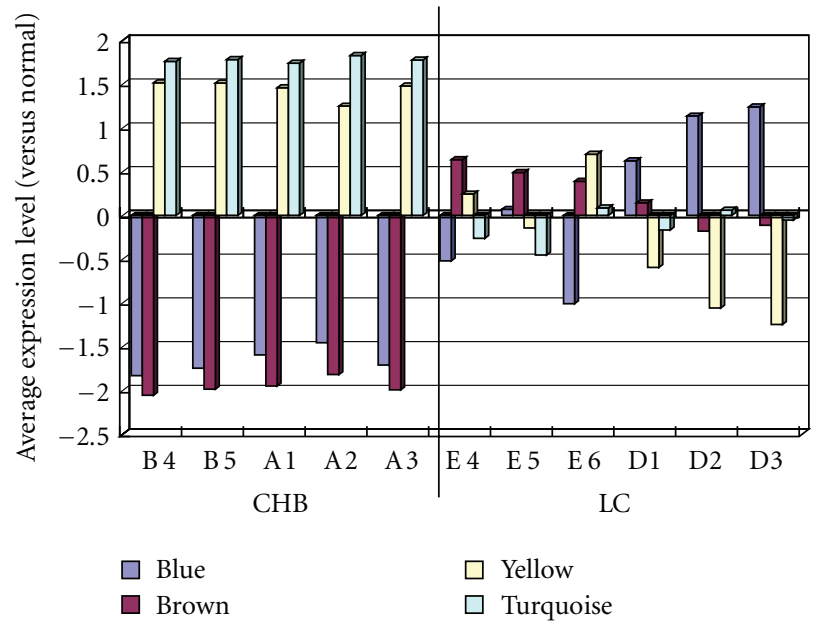

(a)

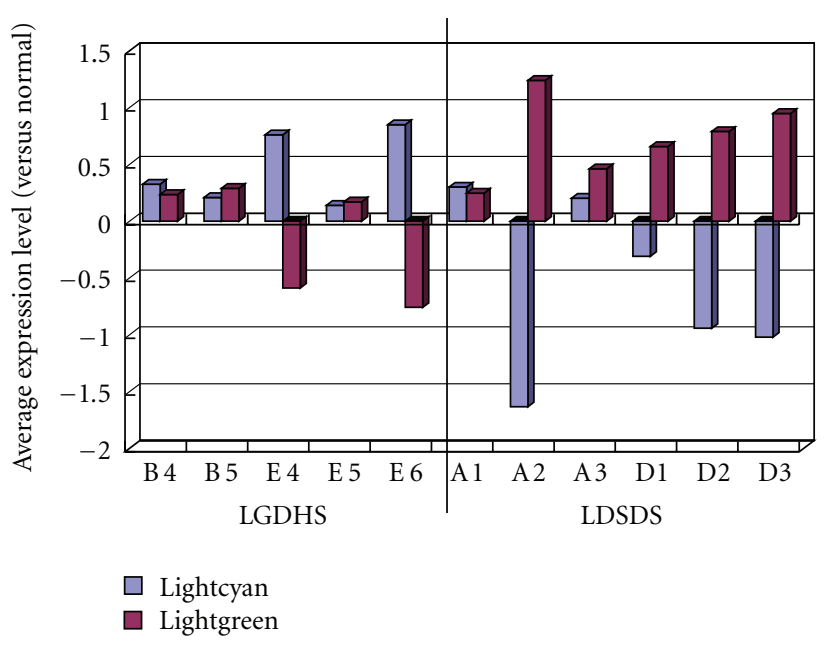

(b)

FIGURE 2: Average gene expression in modules which correlated with diseases or TCM syndromes. In the diseases (a), blue and brown modules both had low expression value in $\mathrm{CHB}$ and not consistent in LC. Yellow and turquoise modules both had high expression value in CHB and not consistent in LC. In the TCM syndromes (b), lightcyan modules had low expression value in LDSDS. Lightgreen modules had high expression value in LDSDS. A1-3 and D1-3: LDSDS; B 4, 5 and E4-6: LGDHS.

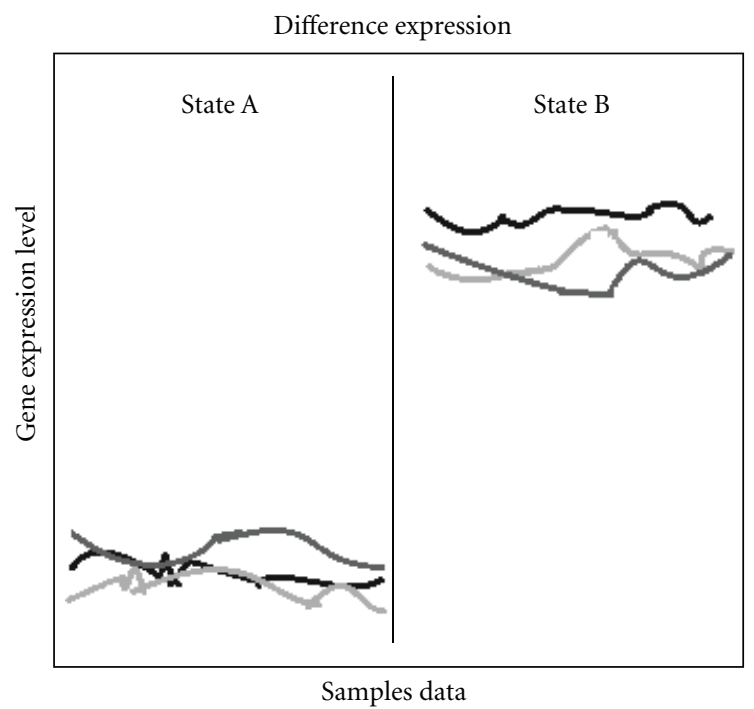

(a)

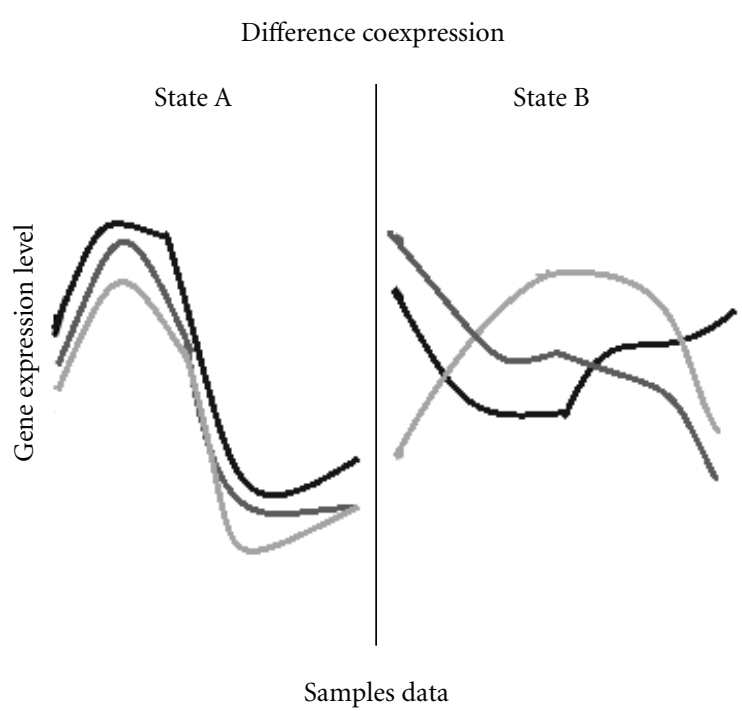

(b)

FIGURE 3: Schematic diagram of difference expression and difference coexpression. Graph of the difference expression (a) represented that there are genes different expression levels between states $\mathrm{A}$ and $\mathrm{B}$, and the difference coexpression (b) represented that there is higher correlation in state A and lower correlation in state B. Curves were represented as whichever genes.

that there was higher gene correlation in a state and lower gene correlation in another state.

Then, the difference coexpression groups between LGDHS and LDSDS were analyzed using the advantage of coXpress R package [16]. First, through the analysis using the 830 differential expression genes $(P<0.05$ in $t$-test $)$ between the LGDHS and LDSDS, the gene groups whose gene members were coexpressed in LGDHS and not coexpressed in LDSDS were produced by coXpress (A in Table 1). Then we also executed the coXpress again to find the gene groups whose gene members were coexpressed in
LDSDS and not coexpressed in LGDHS (B in Table 1). The $P$ values including p.g1 in and p.g2 indicated a gene confusion degree in every group in LGDHS or LDSDS, respectively, $(P>0.05$ was jumbled or not coexpressed; $P<0.05$ was order or coexpressed).

It was found that the gene coexpression groups were orderly in LGDHS but jumbled in LDSDS (A in Table 1). Among the groups jumbled in LDSDS, There were the most gene numbers in group 9. The gene confusion degree in group 9 was showed in Figure 4. It was observed that genes of LGDHS in group 9 had similar traces (Figure 4(a)), whereas 
TABLE 1: Comparison of gene coexpression groups in LGDHS and LDSDS.

\begin{tabular}{|c|c|c|c|}
\hline Group ID & Gene number & P.g1 & P.g2 \\
\hline \multicolumn{4}{|c|}{ A LGDHS } \\
\hline 8 & 6 & 0.00 & 0.62 \\
\hline 5 & 10 & 0.00 & 0.31 \\
\hline 9 & 81 & 0.00 & 0.83 \\
\hline 14 & 18 & 0.00 & 0.38 \\
\hline 12 & 34 & 0.00 & 0.11 \\
\hline 17 & 15 & 0.00 & 0.05 \\
\hline 13 & 45 & 0.00 & 0.14 \\
\hline 10 & 58 & 0.00 & 0.03 \\
\hline 4 & 19 & 0.00 & 0.15 \\
\hline 16 & 27 & 0.00 & 0.02 \\
\hline 15 & 55 & 0.00 & 0.00 \\
\hline 3 & 48 & 0.00 & 0.00 \\
\hline 6 & 16 & 0.00 & 0.01 \\
\hline 11 & 92 & 0.00 & 0.00 \\
\hline 2 & 11 & 0.00 & 0.00 \\
\hline 1 & 234 & 0.00 & 0.00 \\
\hline 7 & 61 & 0.00 & 0.00 \\
\hline \multicolumn{4}{|c|}{ B LDSDS } \\
\hline 9 & 6 & 0.00 & 0.00 \\
\hline 17 & 10 & 0.00 & 0.00 \\
\hline 12 & 13 & 0.01 & 0.00 \\
\hline 7 & 297 & 0.00 & 0.00 \\
\hline 14 & 5 & 0.12 & 0.00 \\
\hline 4 & 90 & 0.00 & 0.00 \\
\hline 8 & 5 & 0.20 & 0.00 \\
\hline 10 & 12 & 0.04 & 0.00 \\
\hline 5 & 69 & 0.53 & 0.00 \\
\hline 6 & 26 & 0.83 & 0.00 \\
\hline 15 & 3 & 0.49 & 0.08 \\
\hline 2 & 238 & 0.69 & 0.00 \\
\hline 3 & 21 & 0.87 & 0.00 \\
\hline 11 & 8 & 0.54 & 0.00 \\
\hline 1 & 8 & 0.36 & 0.00 \\
\hline 13 & 4 & 0.62 & 0.05 \\
\hline 18 & 6 & 0.83 & 0.00 \\
\hline 16 & 9 & 0.76 & 0.07 \\
\hline
\end{tabular}

the traces of LDSDS were varied (Figure 4(b)). To further clarify the functional mechanism at molecular level, GO enrichment analysis was taken on the genes in group 9. As Table 2 revealed, LGDHS was involved in electron transport chain function, but LDSDS does not.

Analogously, it was also found that the gene coexpression groups were orderly in LDSDS but jumbled in LGDHS (B in Table 1). Among the groups jumbled in LGDHS, there were the most gene numbers in group 2. Therefore, group 2 were analyzed and showed that the traces of LGDHS were varied (Figure 4(c)) and the traces of LDSDS were in order (Figure 4(d)). Through further studied the molecular functional mechanism by the GO enrichment analysis, it was found that LDSDS was involved in G-protein-coupled receptor protein-signaling pathway (GCRP pathway), but LGDHS does not (Table 2).

3.4. Molecular Mechanism of Difference between Diseases and TCM Syndromes. It was interesting in our result that the genes coexpression in group 2 was enriched in GCRP pathway. Because same situation happened to the genes in blue module, which was related with the difference between $\mathrm{CHB}$ and LC by the gene module analysis, these genes in GCRP pathway were differentially expressed between $\mathrm{CHB}$ and LC and difference coexpressed between LGDHS and LDSDS. These results were summarized in Figure 5. Interestingly, in GCRP pathway, whether TCM syndrome was LGDHS or LDSDS, the gene expression level was lower in $\mathrm{CHB}$ and higher or lower in LC, and whether disease was CHB or LC, the genes in LDSDS had higher correlation than LGDHS. For example, in LDSDS, genes GPER, PTHR1, GPR173, and SSTR1 were connected in a correlation network together, while they, respectively, belong to four correlation networks in LGDHS (Figure 5). These results suggested the different molecular mechanism between diseases (CHB and LC) and TCM syndromes (LGDHS and LDSDS).

3.5. Average Expression and Correlation of DRD5 GABRA SSTR1 and NPFF Genes in Diseases and TCM Syndromes. To test and verify the difference of average expression level and correlation of genes in GCRP pathway, DRD5 GABRA SSTR1 and NPFF mRNAs were expressed by real-time RT-PCR. The average expression levels of these genes in both LGDHS and LDSDS were lower in CHB, and that of LDSDS was more than LGDHS in LC (Figure 6(a)). The correlation coefficient of LDSDS $(>0.5)$ in CHB and LC was more than LGDHS $(<0.5)$ in CHB and LC (Figure 6(b)). These results further confirmed that the gene expression level was lower in $\mathrm{CHB}$ and higher or lower in LC. The genes in LDSDS had higher correlation than LGDHS whether disease was CHB or LC.

Previous researches had also found that LC was related with GCRP pathway [17-19], but little literature touched upon the relation between CHB and GCRP. Our result also indicated that genes in GCRP pathway were higher expression in LC and lower expression in CHB. It suggested that LC was a more serious disease than CHB by the activity of GCRP pathway. Further research will clarify the role of genes in GCRP pathway from CHB develop to LC.

Interestingly, our results showed that TCM syndromes, LGDHS and LDSDS did not clearly relate with the gene expression levels in GCRP pathway. The genes correlation or cooperation was more important. As shown in Figure 4, the genes in LDSDS had more connections than LGDHS, so LGDHS and LDSDS constructed different gene network. It incarnated the holistic thought in TCM.

Therefore, our research results suggested that $\mathrm{CHB}$ could be divided into LGDHS and LDSDS by the gene correlation as well as LC, which reveals the molecular feature of Different TCM Syndrome for Same Disease. Analogously, LGDHS was being in CHB or LC by the gene expression level as well as LDSDS, which reveals the molecular feature of Same TCM 


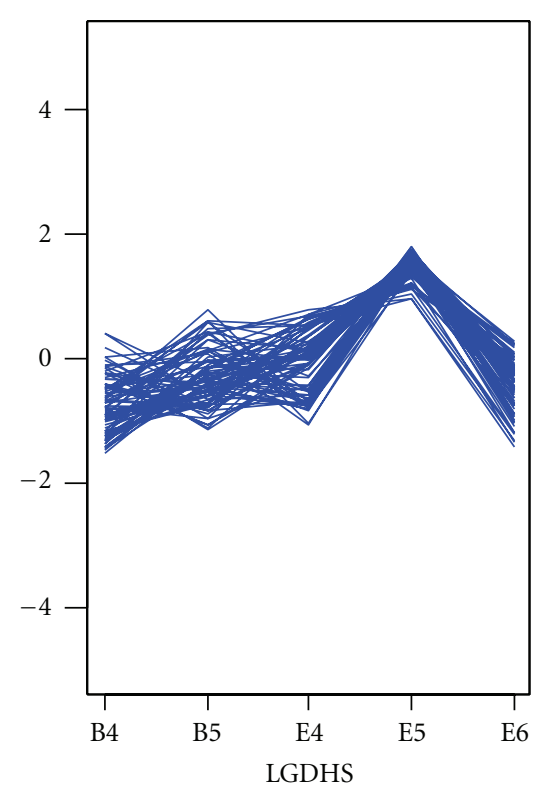

(a)

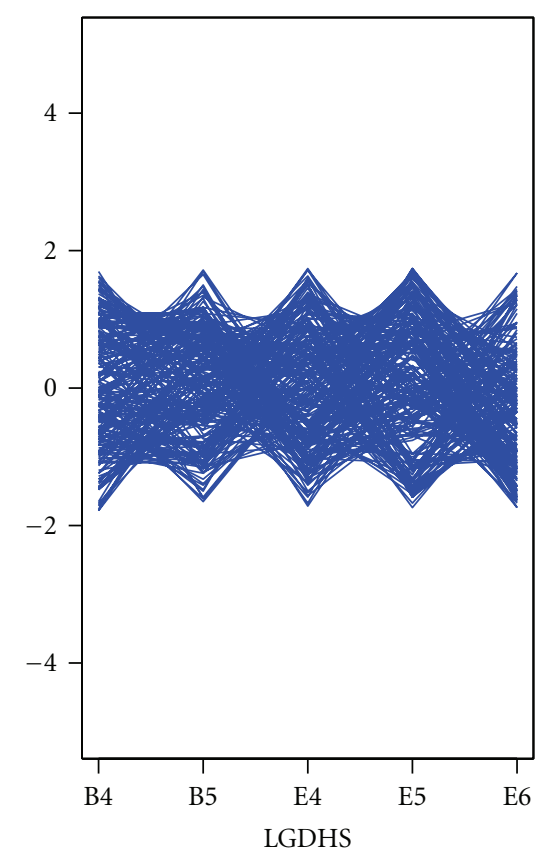

(c)

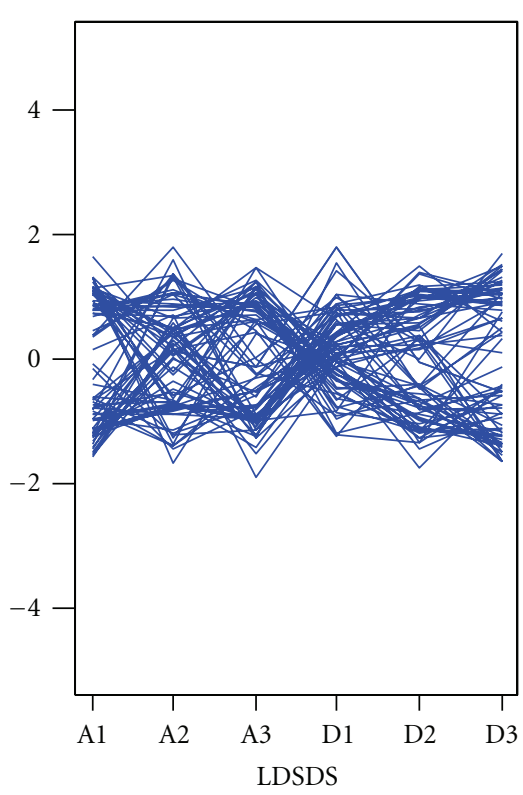

(b)

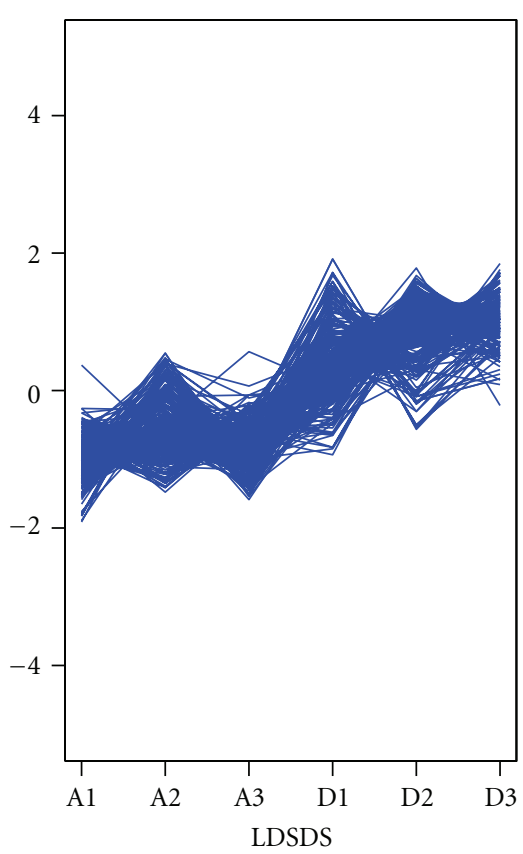

(d)

FIGURE 4: The gene confusion degree of group 2 and 9 in LGDHS and LDSDS. CoXpress was used to find orderly gene groups in LGDHS or LDSDS. The genes in group 9 of orderly gene groups in LGDHS showed good consistency in LGDHS (a) and poor consistency in LDSDS (b). The genes in group 2 of orderly gene groups in LDSDS showed poor consistency in LGDHS (c) and good consistency in LDSDS (d). A1-3 and D1-3: LDSDS; B 4, 5 and E4-6: LGDHS.

Syndrome for Different Diseases. The schematic diagram of the molecular mechanisms was showed in Figure 2.

There are two kinds of therapeutic principles in the TCM syndrome identification and treatment process, called Different treatments for the same disease and same treatment for different diseases. The Different treatments for the same disease means using different prescriptions or Chinese herbal medicines to treat the different TCM syndromes in the same disease process. The Same treatment for different diseases means using the same and prescriptions or Chinese herbal medicines to treat the same TCM syndrome in different disease process. These therapeutic principles are widely used in TCM practice as personalized therapy $[12,20]$. Therefore, understanding the molecular mechanisms of Same TCM Syndrome for Different Diseases and Different TCM Syndrome for Same Disease will be primely serving for TCM 

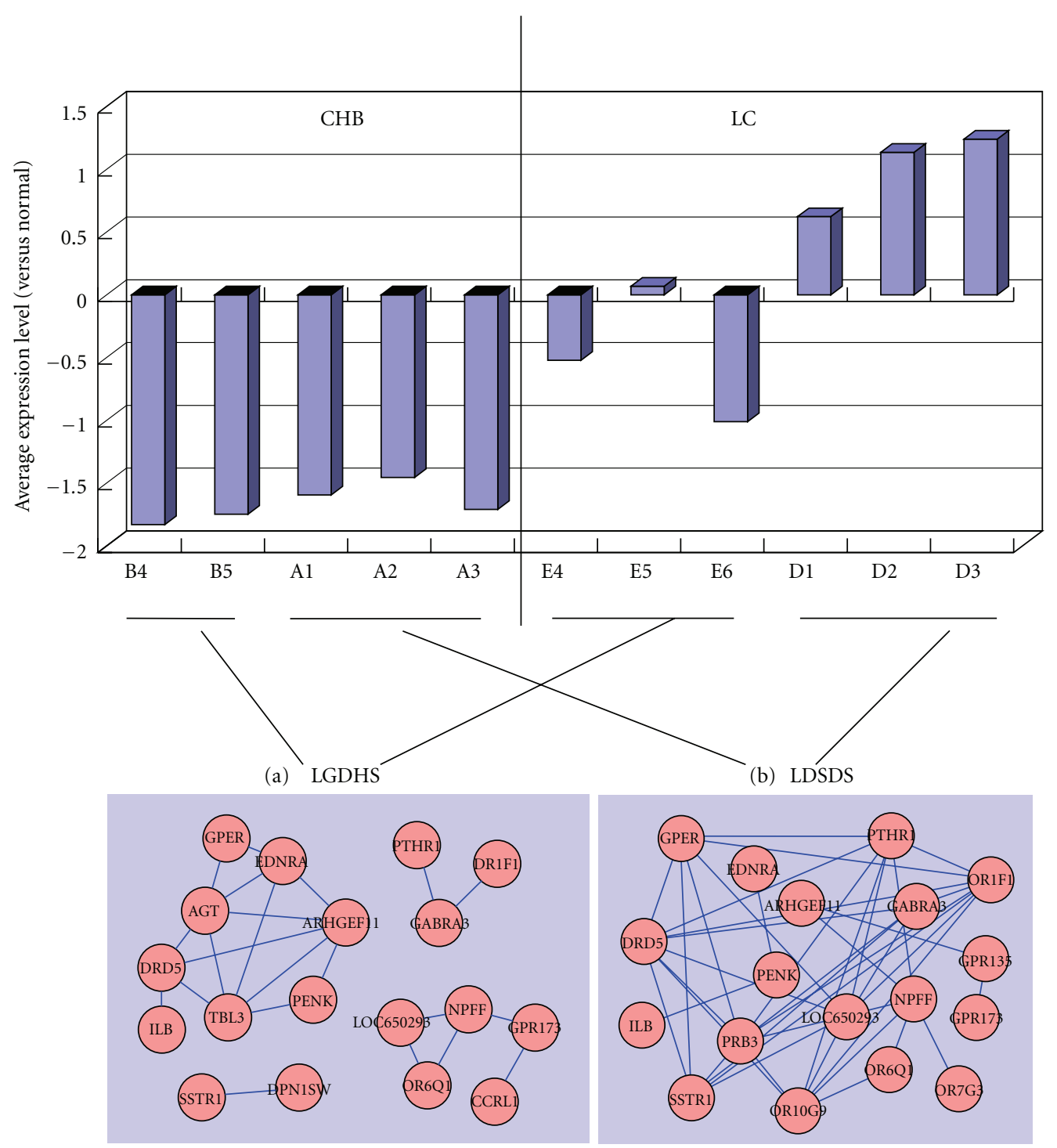

FIGURE 5: Gene relationships in GCRP pathway in diseases and TCM syndromes. GO enrichment analysis of genes in group 2 was carried out. Whether diseases (CHB or LC) and TCM syndromes (LGDHS or LDSDS) were correlated to GCRP pathway, the gene expression (upper figure) was represented that the gene expression levels were lower in CHB and higher or lower in LC. The gene network ((a), (b)) was represented that the genes connections in LDSDS (b) were more than LGDHS (a).

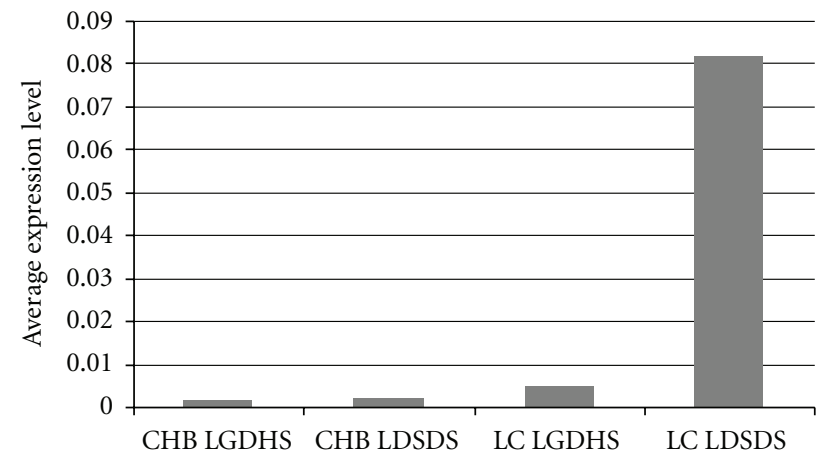

(a)

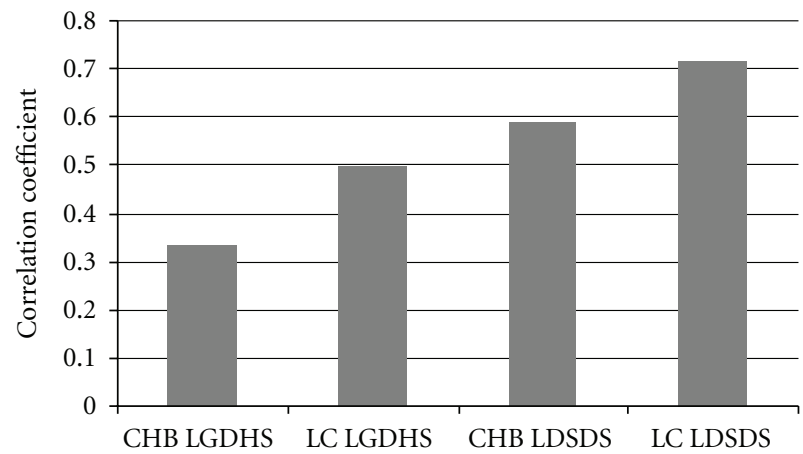

(b)

FIgURE 6: Average expression and correlation of DRD5 GABRA SSTR1 and NPFF mRNAs in diseases and TCM syndromes. The gene expression levels of both LGDHS and LDSDS were lower in CHB and that of LDSDS was more than LGDHS in LC (a). (Gene expression levels were the ratio of each mRNA and ACTB mRNA). The correlation coefficient of LDSDS in CHB and LC was more than LGDHS in CHB and LC (b). 
TABLE 2: GO enrichments of orderly group 2 in LDSDS and group 9 in LGDHS.

\begin{tabular}{|c|c|c|c|}
\hline GO term ID & Orderly group & Enrichment $P$ & Term name \\
\hline GO:0006120 & LGDHS 9 & 0.022478 & Mitochondrial electron transport, NADH to ubiquinone \\
\hline GO:0022900 & LGDHS 9 & 0.022478 & Electron transport chain \\
\hline GO:0022904 & LGDHS 9 & 0.022478 & Respiratory electron transport Chain \\
\hline GO:0042773 & LGDHS 9 & 0.022478 & ATP synthesis coupled electron transport \\
\hline GO:0042775 & LGDHS 9 & 0.022478 & Organelle ATP synthesis coupled electron transport \\
\hline GO:0006119 & LGDHS 9 & 0.04236 & Oxidative phosphorylation \\
\hline GO:0010468 & LGDHS 9 & 0.048855 & Regulation of gene expression \\
\hline GO:0009987 & LGDHS 9 & 0.049535 & Cellular process \\
\hline GO:0016070 & LGDHS 9 & 0.059695 & RNA metabolic process \\
\hline GO:0006355 & LGDHS 9 & 0.061016 & Regulation of transcription, DNA-dependent \\
\hline GO:0007186 & LDSDS2 & 0.000668 & G-protein coupled receptor protein signaling pathway \\
\hline GO:0007606 & LDSDS2 & 0.004518 & Sensory perception of chemical stimulus \\
\hline GO:0007608 & LDSDS2 & 0.004518 & Sensory perception of smell \\
\hline GO:0007166 & LDSDS2 & 0.014079 & Cell surface receptor linked signal transduction \\
\hline GO:0007586 & LDSDS2 & 0.015106 & digestion \\
\hline GO:0007223 & LDSDS2 & 0.017534 & Wnt receptor signaling pathway, calcium modulating pathway \\
\hline GO:0008203 & LDSDS2 & 0.017534 & Cholesterol metabolic process \\
\hline GO:0016125 & LDSDS2 & 0.017534 & Sterol metabolic process \\
\hline GO:0042157 & LDSDS2 & 0.017534 & Lipoprotein metabolic process \\
\hline GO:0006813 & LDSDS2 & 0.017952 & Potassium ion transport \\
\hline
\end{tabular}

diagnosis and treatment. This research provided firstly the evidence. Further research will be required more samples to proving this evidence.

\section{Conclusion}

The classification of TCM syndrome is a diagnostic method. TCM syndromes are significantly associated with diseases, which are involved in Same TCM Syndrome for Different Diseases and Different TCM Syndrome for Same Disease. In this study, through analyzing microarray date of LGDHS and LDSDS in patients with CHB and LC, we provided evidence that the difference between $\mathrm{CHB}$ and LC was gene expression and the difference between LGDHS and LDSDS was gene coexpression in G-protein-coupled receptor protein-signaling pathway. Therein genes GPER, PTHR1, GPR173, and SSTR1 were coexpressed in LDSDS but not in LGDHS. Either CHB or LC was divided into the alternative LGDHS and LDSDS by the gene correlation, which reveals the molecular feature of Different TCM Syndrome for Same Disease. Either LGDHS or LDSDS was divided into the alternative $\mathrm{CHB}$ and LC by the gene expression level, which reveals the molecular feature of Same TCM Syndrome for Different Diseases. These results might be significant for both TCM research and TCM diagnosis and treatment.

\section{Authors' Contribution}

Z. Guo and A. Yu equally contributed in this paper.

\section{Acknowledgments}

This study was supported by National Science and Technology Major Project of China (no. 2012ZX10005001-004 and no. 2009ZX09311-003), Leading Academic Discipline Project of Shanghai Municipal Education Commission (no. J50301), and E-institutes of Shanghai Municipal Education Commission (no. E 03008).

\section{References}

[1] W. Jia, W. Y. Gao, Y. Q. Yan et al., "The rediscovery of ancient Chinese herbal formulas," Phytotherapy Research, vol. 18, no. 8, pp. 681-686, 2004.

[2] A. P. Lu and K. J. Chen, "Integrative medicine in clinical practice: from pattern differentiation in traditional Chinese medicine to disease treatment," Chinese Journal of Integrative Medicine, vol. 15, no. 2, p. 152, 2009.

[3] Y. H. Lu, H. P. Hao, G. J. Wang, X. H. Chen, X. X. Zhu, and B. R. Xiang, "Metabolomics approach to the biochemical differentiation of Traditional Chinese Medicine syndrome types of hypertension," Chinese Journal of Clinical Pharmacology and Therapeutics, vol. 12, no. 10, pp. 1144-1150, 2007.

[4] W. Jian, Z. Yuan, X. Huang et al., "Analysis on urine metabolomics of coronary heart disease patients with the heart blood stasis syndrome," Journal of Traditional Chinese Medicine, vol. 51, no. 8, pp. 729-732, 2010.

[5] C. Lu, C. Xiao, G. Chen et al., "Cold and heat pattern of rheumatoid arthritis in traditional Chinese medicine: distinct molecular signatures indentified by microarray expression profiles in CD4-positive T cell," Rheumatology International, vol. 32, no. 1, pp. 61-68, 2010. 
[6] S. Li, Z. Q. Zhang, L. J. Wu, X. G. Zhang, Y. D. Li, and Y. Y. Wang, "Understanding ZHENG in traditional Chinese medicine in the context of neuro-endocrine-immune network," IET Systems Biology, vol. 1, no. 1, pp. 51-60, 2007.

[7] WHO Media Centre and B. Hepatitis, http://www.who.int/ mediacentre/factsheets/fs204.

[8] X. Cui, Y. Wang, N. Kokudo, D. Fang, and W. Tang, "Traditional Chinese medicine and related active compounds against hepatitis B virus infection," Bioscience Trends, vol. 4, no. 2, pp. 39-47, 2010.

[9] . C. Liu, Y. Hu, L. Xu, and P. Liu, "Effect of Fuzheng Huayu formula and its actions against liver fibrosis," Chinese Medicine, vol. 4, p. 12, 2009.

[10] Z. Y. Li, X. X. Zhang, and Z. C. Xu, "Study on integrative point of traditional and western medicine-from "integrative disease and syndrome" to "integrative pathological process and syndrome"," Zhongguo Zhong Xi Yi Jie He Za Zhi, vol. 25, no. 3, pp. 259-262, 2005.

[11] X. Q. Li, H. Zhang, and X. W. Li, "Study on reference laboratory diagnostic index of liver-fire ascending syndrome," Zhongguo Zhong Xi Yi Jie He Za Zhi, vol. 21, no. 3, pp. 190-192, 2001.

[12] J. Li, "Thinking on syndrome differentiation treatment and personalized therapy for tumor," Zhong Xi Yi Jie He Xue Bao, vol. 7, no. 4, pp. 306-308, 2009.

[13] X. X. Zeng, Z. X. Bian, T. X. Wu, S. F. Fu, E. Ziea, and W. T. Woon, "Traditional chinese medicine syndrome distribution in chronic hepatitis B populations: a systematic review," The American Journal of Chinese Medicine, vol. 39, no. 6, pp. 10611074, 2011.

[14] Y. A. Ye, F. Jiang, Z. M. Zhao et al., "Chinese medical pattern distribution of chronic type hepatitis B," Journal of Traditional Chinese Medicine, vol. 48, no. 3, pp. 256-258, 2007.

[15] P. Langfelder and S. Horvath, "WGCNA: an R package for weighted correlation network analysis," BMC Bioinformatics, vol. 9, article 559, 2008.

[16] M. Watson, "CoXpress: differential co-expression in gene expression data," BMC Bioinformatics, vol. 7, article 509, 2006.

[17] T. Y. Chen, T. L. Hwang, C. Y. Lin et al., "EMR2 receptor ligation modulates cytokine secretion profiles and cell survival of lipopolysaccharide-treated neutrophils," Chang Gung Medical Journal, vol. 34, no. 5, pp. 468-477, 2011.

[18] C. Rancoule, J. P. Pradère, J. Gonzalez et al., "Lysophosphatidic acid-1-receptor targeting agents for fibrosis," Expert Opinion on Investigational Drugs, vol. 20, no. 5, pp. 657-667, 2011.

[19] H. E. Wasmuth and R. Weiskirchen, "Pathogenesis of liver fibrosis: modulation of stellate cells by chemokines," Zeitschrift fur Gastroenterologie, vol. 48, no. 1, pp. 38-45, 2010.

[20] Y. Liang, Z. Lu, N. Zhang, and L. Shen, "Evaluation of multidimensional outcomes of chronic diseases: a clinical example from China," Archives of Gerontology and Geriatrics, vol. 52, no. 3, pp. e106-e109, 2011. 


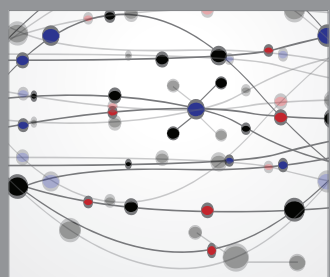

The Scientific World Journal
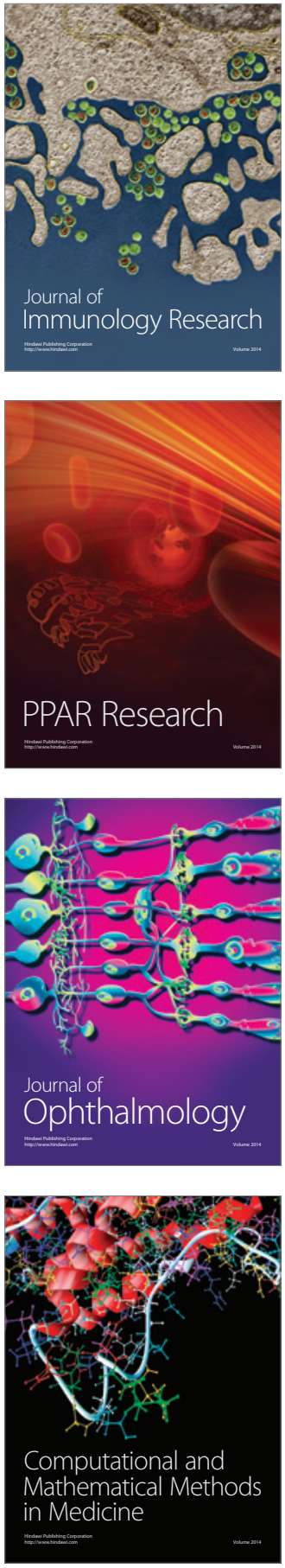

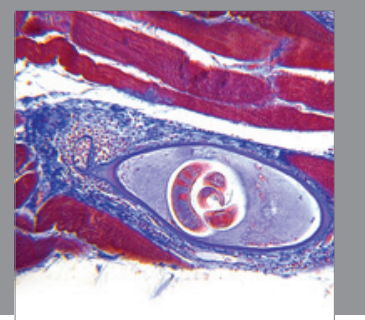

Gastroenterology

Research and Practice
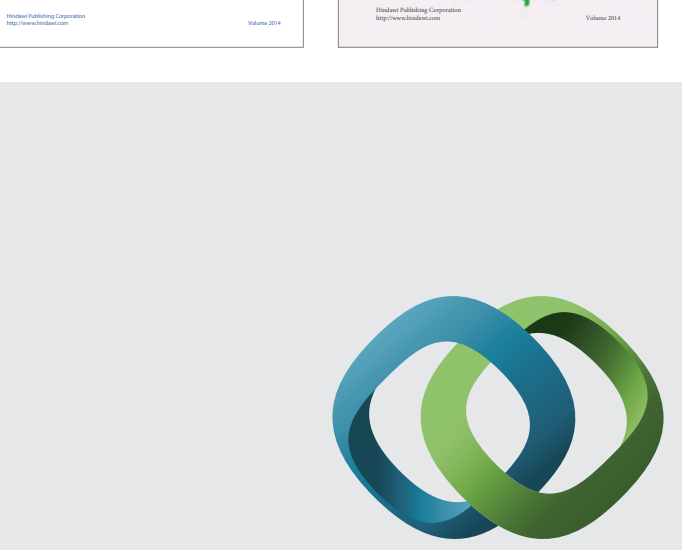

\section{Hindawi}

Submit your manuscripts at

http://www.hindawi.com
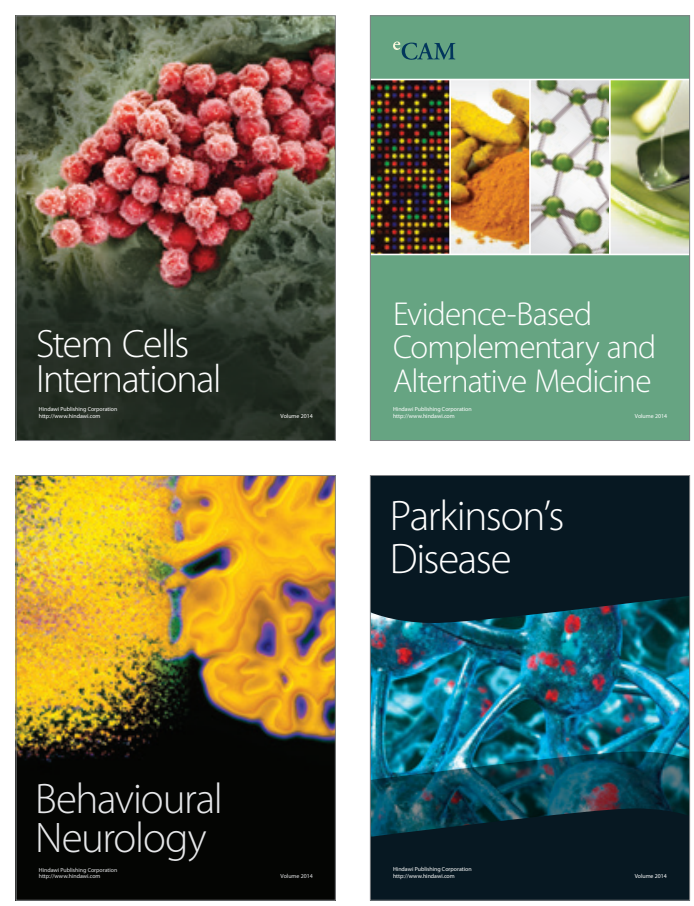

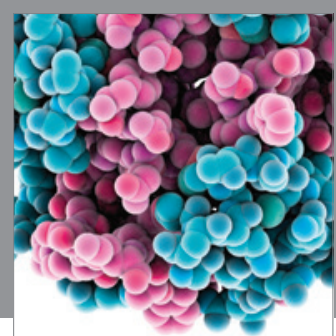

Journal of
Diabetes Research

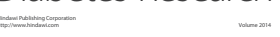

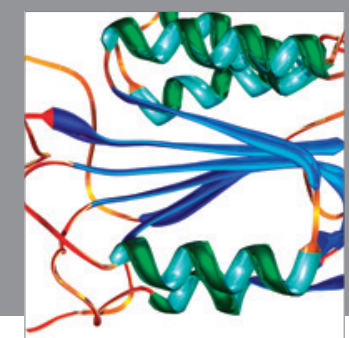

Disease Markers
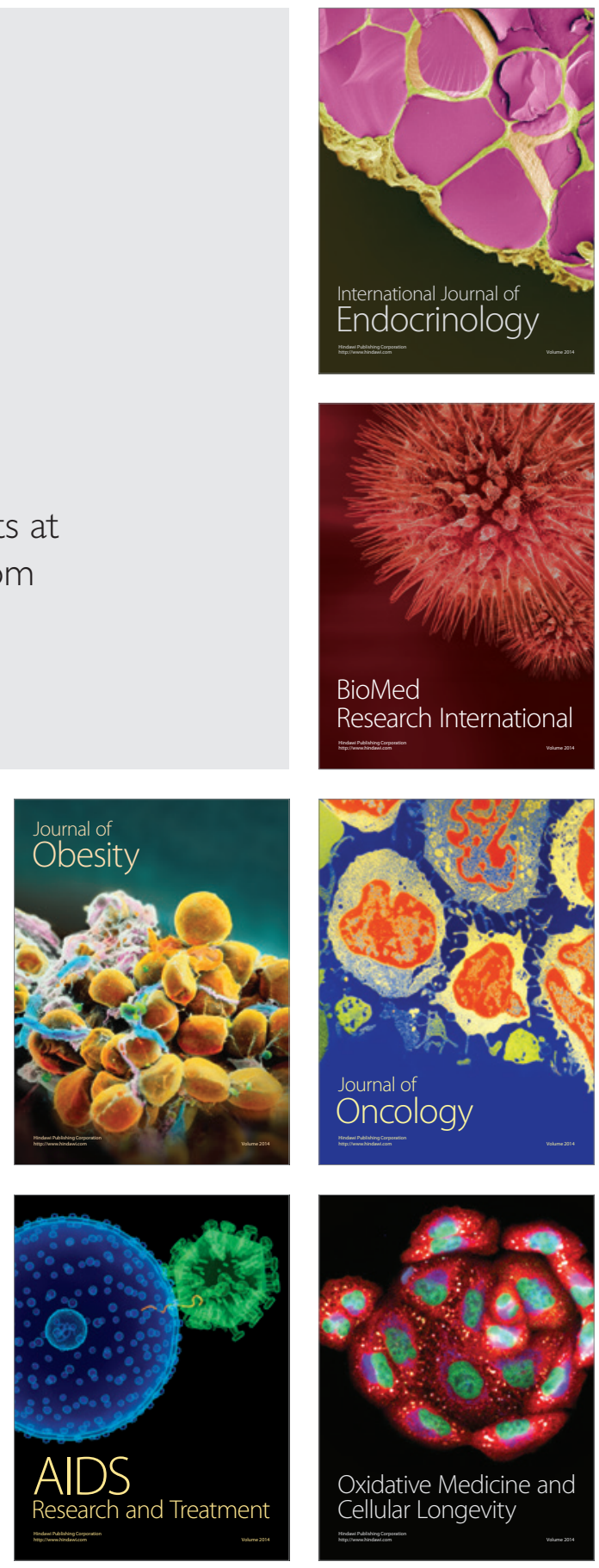